\title{
THE BOUNDEDNESS OF THE SOLUTIONS OF A NONLINEAR DIFFERENTIAL EQUATION
}

\author{
CHOY-TAK TAAM
}

1. Introduction. In this note we consider the differential equation

$$
\left(r(x) y^{\prime}\right)^{\prime}+q(x) y=f(x, y)
$$

with a "small" nonlinear term $f(x, y)$. The exact conditions satisfied by $r, q$, and $f$ will be given in $\S \S 2$ and 3 .

If $r, q$, and $(r q)^{\prime}$ are real-valued continuous functions on the positive $x$-axis, in a recent paper [1] Walter Leighton proved that the following conditions $r q>0$ and $(r q)^{\prime} \geqq 0$ for $x \geqq 0$ are sufficient for the boundedness of the solutions of the linear differential equation $\left(r y^{\prime}\right)^{\prime}+q y=0$ on the positive $x$-axis. It is the purpose of this note to point out that, even if $r q$ is not differentiable (in fact, even the continuity of $r q$ is not required), under certain suitable conditions satisfied by $r, q$, and $f$ as stated in Theorems 1 and 2, we still can test the boundedness of the solutions of (1) by a method which includes Leighton's method as a special case. In $\$ 2$ we assume that $r, q$, and $f$ are real-valued functions; in $\S 3$, complex-valued.

2. Boundedness of the solutions. Throughout this section we assume that $1 / r(x), q(x)$, and $f(x, y)$ (for each fixed $y$ ) are real-valued functions defined for all $x \geqq 0$ and belonging to $L(0, R)$ for every positive $R$. Furthermore we assume that for each $x \geqq 0, f(x, y)$ is a continuous function of $y$ satisfying the Lipschitz condition

$$
\left|f\left(x, y_{1}\right)-f\left(x, y_{2}\right)\right| \leqq g(x)\left|y_{1}-y_{2}\right|,
$$

where $g(x)$ and $f(x, 0)$ belong to $L(0, \infty)$.

By a solution of (1) we mean a function $y(x)$ which is absolutely continuous and $r(x) y^{\prime}(x)$ is equal to an absolutely continuous function $z(x)$, say, almost everywhere such that, with $z(x)$ replacing $r(x) y^{\prime}(x),(1)$ is satisfied almost everywhere on $0 \leqq x<\infty$. For the existence and uniqueness of solutions, see [2, sections 68.3 and 68.5].

Denoting $\max \left(u^{\prime}(x), 0\right)$ by $(u(x))_{+}^{\prime}$ and $\min \left(u^{\prime}(x), 0\right)$ by $(u(x))_{-}^{\prime}$, the theorem we shall establish is

THEOREM 1. If there exists a real-valued function $p=p(x)$ such that 1. $p$ belongs to $L(0, R)$ for every positive $R$,

Presented to the Society, December 28, 1953 under the title The boundedness of the solutions of an almost linear differential equation; received by the editors June 29, 1953. 
2. $r p$ is positive and absolutely continuous on $0 \leqq x<\infty$,

3. $p-q$ and $(r p)^{-1}(r p)^{\prime}$ - belong to $L(0, \infty)$, then every solution of (1) is bounded on $0 \leqq x<\infty$.

Proof. First we prove that every solution of

$$
\left(r(x) y^{\prime}\right)^{\prime}+p(x) y=0
$$

is bounded on $0 \leqq x<\infty$. This can be done by modifying Leighton's proof [1]. Multiplying (3) by $r y^{\prime}$ and integrating from 0 to $x$, we obtain

$$
\left(r y^{\prime}\right)^{2}+r p y^{2}=c+\int_{0}^{x}(r p)^{\prime} y^{2} d x
$$

where $c$ is a positive constant. From (4), clearly,

$$
r p y^{2} \leqq c+\int_{0}^{x}(r p)_{+}^{\prime} y^{2} d x, \quad x \geqq 0 .
$$

Multiplying both sides of (5) by $(r p)^{-1}(r p)_{+}^{\prime}$ and then dividing by $c+\int_{0}^{x}(r p)_{+}^{\prime} y^{2} d x$, we have

$$
\frac{(r p)_{+}^{\prime} y^{2}}{c+\int_{0}^{x}(r p)_{+}^{\prime} y^{2} d x} \leqq(r p)^{-1}(r p)_{+}^{\prime}, \quad x \geqq 0 .
$$

Integration of (6) from 0 to $x$ gives

$$
\log \left(c+\int_{0}^{x}(r p)_{+}^{\prime} y^{2} d x\right) \leqq \log c+\int_{0}^{x}(r p)_{+}^{\prime}(r p)^{-1} d x, \quad x \geqq 0 .
$$

(5) and (7) then yield

$$
r p y^{2} \leqq c \exp \left(\int_{0}^{x}(r p)_{+}^{\prime}(r p)^{-1} d x\right)
$$$$
x \geqq 0 \text {. }
$$

Since $(r p)_{+}^{\prime}=(r p)^{\prime}-(r p)_{-}^{\prime}$, a simple calculation shows that

$$
r(0) p(0) y^{2} \leqq c \exp \left(-\int_{0}^{x}(r p)_{-}^{\prime}(r p)^{-1} d x\right), \quad x \geqq 0 .
$$

In view of the convergence of the integral in (9), $y$ is bounded on $0 \leqq x<\infty$.

To prove the boundedness of the solutions of (1), we consider the integral equation 
(10)

$$
y(x)=A z_{1}(x)+B z_{2}(x)+\int_{0}^{x} h(t, y(t))\left[z_{2}(x) z_{1}(t)-z_{1}(x) z_{2}(t)\right] d t,
$$

where

$$
h(x, y)=(p(x)-q(x)) y+f(x, y)
$$

and $z_{1}(x)$ and $z_{2}(x)$ are two linearly independent solutions of (3) with

$$
r(x)\left[z_{1}(x) z_{2}^{\prime}(x)-z_{1}^{\prime}(x) z_{2}(x)\right]=1
$$

almost everywhere and $A$ and $B$ are arbitrary constants. Clearly $h(x, y)$ satisfies

$$
\left|h\left(x, y_{1}\right)-h\left(x, y_{2}\right)\right| \leqq k(x)\left|y_{1}-y_{2}\right|,
$$

where $k(x)=|p(x)-q(x)|+g(x)$, and $k(x)$ and $h(x, 0)=f(x, 0)$ belong to $L(0, \infty)$. Obviously (11) implies that $|h(x, y)| \leqq k(x)|y|$ $+|h(x, 0)|$, and so the integral in (10) exists for every continuous function $y(x)$. We now show that (10) has a solution by successive approximations. Define $y_{0}(x)=0$ and

$$
\begin{gathered}
y_{n}(x)=A z_{1}(x)+B z_{2}(x) \\
+\int_{0}^{x} h\left(t, y_{n-1}(t)\right)\left[z_{2}(x) z_{1}(t)-z_{1}(x) z_{2}(t)\right] d t
\end{gathered}
$$

for $n=1,2,3, \cdots$. If $\left|z_{2}(x) z_{1}(t)-z_{1}(x) z_{2}(t)\right| \leqq N$ for $x \geqq 0$ and $t \geqq 0$ and if $M$ is an upper bound of $\left|y_{1}(x)\right|$ on $0 \leqq x<\infty$, using (12) it is easy to verify that

$$
\left|y_{n+1}(x)-y_{n}(x)\right| \leqq M \frac{[H(x)]^{n}}{n !}, \quad H(x)=N \int_{0}^{x} k(t) d t,
$$

for $n=0,1,2, \cdots$. From this it follows that $y_{n}(x)$ converges to a limit $y(x)$ uniformly on $0 \leqq x \leqq x_{0}$ for every $x_{0}$, and $y(x)$ satisfies (10) and

$$
|y(x)| \leqq M \exp (H(x)), \quad x \geqq 0 .
$$

The boundedness of $y(x)$ then follows from the convergence of $H(x)$ and (14). Substitution of (10) into (1) shows that $y(x)$ satisfies (1) and so is a solution of (1). From the uniqueness of the solutions of (1), it follows that for different constants $A$ and $B, y(x)$ represents all the solutions of (1). This proves that every solution of (1) is bounded on $0 \leqq x<\infty$.

We may also observe that, in view of the boundedness of $y(x)$, (10) can be written in the form 


$$
\begin{aligned}
y(x)= & c_{1} z_{1}(x)+c_{2} z_{2}(x) \\
& -\int_{x}^{\infty} h(t, y(t))\left[z_{2}(x) z_{1}(t)-z_{1}(x) z_{2}(t)\right] d t,
\end{aligned}
$$

the integral part approaching zero as limit as $x$ approaches $\infty$. Hence to each solution $y(x)$ of (1), there corresponds a solution $c_{1} z_{1}(x)$ $+c_{2} z_{2}(x)$ of (3) such that their difference tends to zero as $x$ tends to $\infty$. In fact the correspondence is one-to-one. This can be established by proving also the existence and uniqueness of the solution of (15) for two arbitrary constants $c_{1}$ and $c_{2}$. Differentiation of (15) also yields

$$
y^{\prime}(x)=\left(c_{1}+\epsilon_{1}(x)\right) z_{1}^{\prime}(x)+\left(c_{2}+\epsilon_{2}(x)\right) z_{2}^{\prime}(x)
$$

for almost all $x$ on $0 \leqq x<\infty$, where $\epsilon_{1}(x)$ and $\epsilon_{2}(x)$ tend to zero as $x$ tends to $\infty$.

3. Extension. In this section we assume that $r(x), q(x)$, and $f(x, y)$ satisfy the same conditions as stated in the first paragraph of $\$ 2$ except that they are complex-valued functions. Denoting by $a^{*}$ the conjugate of $a$, Theorem 1 can be slightly extended to

THEOREM 2. If there exists a complex-valued function $p=p(x)$ such that

1. $p$ belongs to $L(0, R)$ for every positive $R$,

2. $r p^{*}$ is real, positive and absolutely continuous on $0 \leqq x<\infty$,

3. $p-q$ and $\left(r p^{*}\right)^{-1}\left(r p^{*}\right)_{-}^{\prime}$ belong to $L(0, \infty)$, then every solution of (1) is bounded on $0 \leqq x<\infty$.

Proof. Multiplying (3) by $\left(r y^{\prime}\right)^{*}$ and its conjugate by $r y^{\prime}$, we have the sum

$$
\left(r y^{\prime}\right)^{*}\left(r y^{\prime}\right)^{\prime}+\left(r y^{\prime}\right)\left(r y^{\prime}\right)^{\prime *}+r^{*} p y y^{\prime *}+r p^{*} y^{*} y^{\prime}=0 .
$$

Integration of (17) from 0 to $x$ gives

$$
\left|r y^{\prime}\right|^{2}+r p^{*}|y|^{2}=c+\int_{0}^{x}\left(r p^{*}\right)^{\prime}|y|^{2} d x
$$

where $c$ is a positive constant. The rest of the proof is similar to that of Theorem 1. The remarks at the end of $\$ 2$ also apply here.

\section{REFERENCES}

1. Walter Leighton, Bounds for the solutions of a second-order linear differential equation, Proc. Nat. Acad. Sci. U.S.A. vol. 35 (1949) pp. 190-191.

2. Edward J. McShane, Integration, Princeton, 1947.

The Catholic University of America 